\title{
DFT study on electronic and optical properties of graphene under an external electric field
}

\author{
Abdelhafid Najim ${ }^{1 *}$, Omar Bajjou ${ }^{2}$, Mustapha Boulghallat ${ }^{1}$, Khalid Rahmani ${ }^{3}$ and Lhouceine Moulaoui ${ }^{3}$. \\ ${ }^{1}$ LDD, Faculty of Sciences and Technics, Sultan Moulay Slimane University, BP 523, 23000 Morocco \\ ${ }^{2}$ Material Physics Laboratory, Faculty of Sciences and Technics, Sultan Moulay Slimane University, BP 523, 23000 Beni Mellal, \\ Morocco
}

${ }^{3}$ ERPTM, Polydisciplinary Faculty, Sultan Moulay Slimane University, B.P 592, 23000 Beni Mellal, Morocco

\begin{abstract}
The paper investigates the electronic and optical properties of graphene, under the external electric field $\left(E_{\text {ext }}\right)$ according to perpendicular direction, using density functional theory $(D F T)$. Applying the $E_{\text {ext }}$ to the graphene sheet modifies its electronic and optical properties, including the band gap energy, total density of states (TDOS), absorption coefficient, dielectric function, and refractive index. Graphene's band gap is opened by the application of $E_{\text {ext }}$ to its structure. As a result of the effect of $E_{\text {ext }}$ on graphene layer, its absorption coefficient increases in the ultraviolet $(U V)$ range and decreases in the visible range. We found that the electronic and optical properties of graphene material, can be altered by a perpendicular excitation applied to its structure.
\end{abstract}

\section{Introduction}

Graphene is a semimetal that has zero band gap at the Dirac point. As a member of the 2D-family, it has attracted attention in numerous fields due to its remarkable physical properties. In graphene, the electronic states can be well described by the tight binding Hamiltonian of $\pi$ electrons in carbon atoms [2]. In the carbon atoms of graphene sheets, three atomic orbitals, $2 \mathrm{~s}, 2 \mathrm{p}_{\mathrm{x}}$, and $2 \mathrm{p}_{\mathrm{y}}$ are hybridized to form three $\mathrm{sp}^{2}$ hybrid orbitals, and the $2 \mathrm{p}_{\mathrm{z}}$ orbital remains perpendicular to the other orbitals. In the hybridized orbitals there are three $\sigma$ bonds between the adjacent carbon atoms, and the $2 p_{z}$ orbital results in the $\pi$ bonds. In addition, the nearly $\mathrm{sp}^{2}$ hybridized carbon in the graphene layer supports good electronic conductivity. Therefore, the importance of graphene materials has promoted major research in various fields, such as optoelectronic devices [3].

The electronic and optical properties of graphene have received great attention from a number of applied researches. Applying an electric field on material will cause electro-optic effects and change its optical and electronic properties $[4,5]$. In this paper, first-principles calculations are used to study the influence of $E_{\text {ext }}$ applied on graphene sheet in z-direction on its electronic structure and optical properties, including the optical properties, band gap and TDOS, using CASTEP software.

In the case of applying $E_{\text {ext }}$ to graphene material, the perturbation of the Hamiltonian of $\pi$ electrons by electrostatic potential is expected. The effect of $E_{\text {ext }}$ in

\footnotetext{
* Corresponding author: najim.phymo@gmail.com
}

z-direction of the graphene layer leads to the modification of the Hamiltonian $H$ of the system to:

$$
H=H_{0}+e E_{z}
$$

Where $H_{0}$ is the Hamiltonian of system without external effects.

Our paper is summarized as follows: In Section 2, we briefly introduce the calculation method. Section 3 specifically discusses the numerical results and gives our interpretations. Finally, our research conclusions are included in Section 4.

\section{Computational Methods}

The electronic structure and optical properties simulations of graphene layer, are determined by the $D F T$ calculation. All calculations were performed using the CASTEP code by OTFG ultrasoft pseudopotentials $[6,7]$. The exchange-correlation energy was determined using the Perdew-Burke-Ernzerhof $(P B E)$ functional, within the generalized gradient approximation (GGA) [8]. Throughout all calculations, a plane-wave energy cut-off was set at $500 \mathrm{eV}$. In order to optimize the geometry of graphene sheet, the K-point of the Brillouin zone was sampled by using a $5 \times 5 \times 1$ gamma-centered Monkhorst-Pack grid [9]. In all structural relaxations, the convergence tolerance criteria for energy were set to $2 \times 10^{-6} \mathrm{eV} /$ atom during all geometry optimizations. We optimized carbon atom positions during the atomic relaxation until the force on each atom was less than $0.05 \mathrm{eV} / \AA$, and $0.03 \AA$ for displacement. We set the self- 
consistent field convergence tolerance $(S C F)$ at $2 \times 10^{-6}$ $\mathrm{eV} /$ atom. The maximum stress is set at $0.1 \mathrm{GPa}$.

In this work, the graphene structure was simulated using the model shown in Fig.1. A supercell contains 16 $\mathrm{C}$ atoms, whose coordinates are shown in Tab.1. The graphene structure was placed inside a unit cell of parameters $a=4.919 \AA, b=8.519 \AA, c=3.4 \AA$ and $\alpha=\beta=$ $\gamma=90^{\circ}$ in order to perform an optimization calculation.

Tab. 1 Coordinates of $\mathrm{C}$ atoms in the supercell of graphene.

\begin{tabular}{|c|c|c|c|c|}
\hline \multirow{2}{*}{ Element } & \multirow{2}{*}{$\begin{array}{c}\text { Atom } \\
\text { Number }\end{array}$} & \multicolumn{3}{|c|}{ Fractional coordinates of } \\
\cline { 3 - 5 } & 1 & $\mathrm{u}$ & $\mathrm{v}$ & $\mathrm{w}$ \\
\hline $\mathrm{C}$ & 2 & 0.1249 & 0.1666 & 0.5000 \\
\hline $\mathrm{C}$ & 3 & 0.1249 & 0.4166 & 0.5000 \\
\hline $\mathrm{C}$ & 4 & 0.3750 & 0.0833 & 0.4999 \\
\hline $\mathrm{C}$ & 5 & 0.6250 & 0.1666 & 0.5000 \\
\hline $\mathrm{C}$ & 6 & 0.8749 & 0.4166 & 0.5000 \\
\hline $\mathrm{C}$ & 7 & 0.6250 & 0.3333 & 0.5000 \\
\hline $\mathrm{C}$ & 8 & 0.8749 & 0.0833 & 0.4999 \\
\hline $\mathrm{C}$ & 9 & 0.1249 & 0.6666 & 0.5000 \\
\hline $\mathrm{C}$ & 10 & 0.3750 & 0.9166 & 0.5000 \\
\hline $\mathrm{C}$ & 11 & 0.1249 & 0.8333 & 0.4999 \\
\hline $\mathrm{C}$ & 12 & 0.3750 & 0.5833 & 0.5000 \\
\hline $\mathrm{C}$ & 13 & 0.6250 & 0.6666 & 0.5000 \\
\hline $\mathrm{C}$ & 14 & 0.8749 & 0.9166 & 0.5000 \\
\hline $\mathrm{C}$ & 15 & 0.6250 & 0.8333 & 0.5000 \\
\hline $\mathrm{C}$ & 16 & 0.8749 & 0.5833 & 0.4999 \\
\hline $\mathrm{C}$ & & & &
\end{tabular}

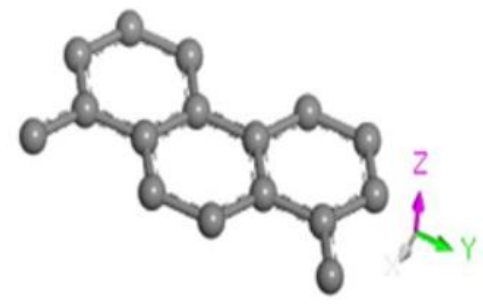

Fig. 1. Crystal structure of graphene. The gray sphere represents carbon atoms.

\section{Results and discussions}

\subsection{Electronic structure}

\subsubsection{Optical gap}

The energy band gap of 2D-materials can be controlled by using an $E_{\text {ext }}$. The band structure of the graphene layer calculated along the direction of high symmetry in the Brillouin zone is plotted in Fig.2. The band structure shows that the minimum value of the conduction band and the maximum value of the valence band are located at $\mathrm{G}$ point in the Brillouin zone, indicating that the band gap of graphene is zero [10]. When $E_{\text {ext }}$ increases from $0 \mathrm{~V} / \AA$ to $0.4 \mathrm{~V} / \AA$, the band gap of graphene increases from $0 \mathrm{eV}$ to $4.028 \mathrm{eV}$. The response of graphene's band gap to the influence of $E_{\text {ext }}$ applied in z-direction promotes the modulation of this electronic property. It can be seen that the inclusion of $E_{\text {ext }}$ changes the energy level of graphene material. Therefore, under the $E_{\text {ext }}$ effect, the band gap indicates the semiconductor properties of graphene. Applied a perturbation potential on graphene will break the symmetry and cause the mixing of its electronic states. The magnitude of $E_{\text {ext }}$ should cause a significant modulation of the band gap of graphene. The results obtained show the opening of the forbidden band as a function of the strength of $E_{\text {ext }}$ in zdirection. This makes graphene a material with controllable band gap energy, which can open the door to the development of photodetectors and lasers controlled by $E_{\text {ext }}$ effect.
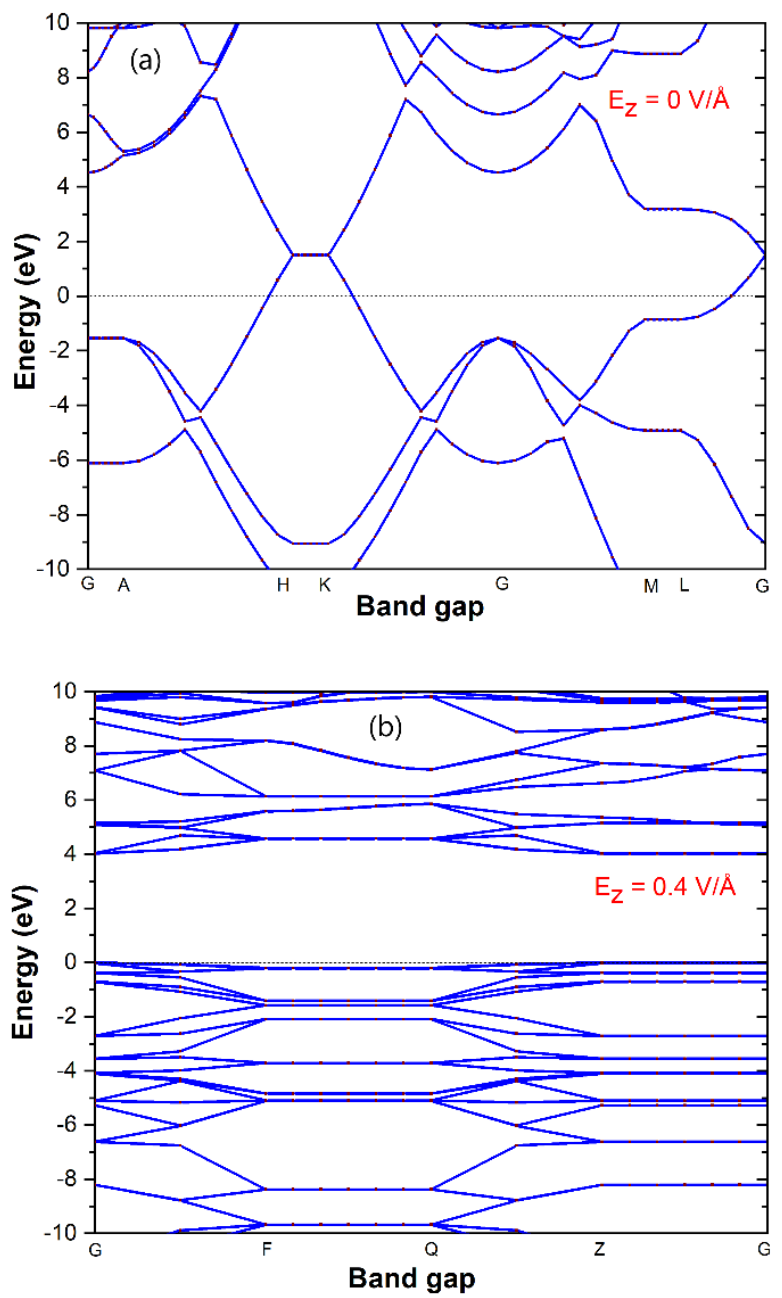

Fig. 2. Graphene energies band gap under external electric field applied in z-direction.

\subsubsection{Density of stats}

Most of the properties of crystals, including their optical and electronic properties, are determined by their TDOS. The description of these states defines the conductive properties of the crystal. The TDOS of the graphene layer is plotted as a function of $E_{\text {ext }}$ applied to zdirection, as shown in Fig.3. The state of all atoms in the 
graphene structure near the Fermi level is mainly derived from the $2 p_{z}$ orbital of C $2 p$. Due to the increase of band gap energy, the probability of electrons for occupying near the Fermi level is reduced by reason of the influence of $E_{\text {ext }}$. These results illustrate the strong effects of $E_{\text {ext }}$ on the electronic properties of graphene material [11].

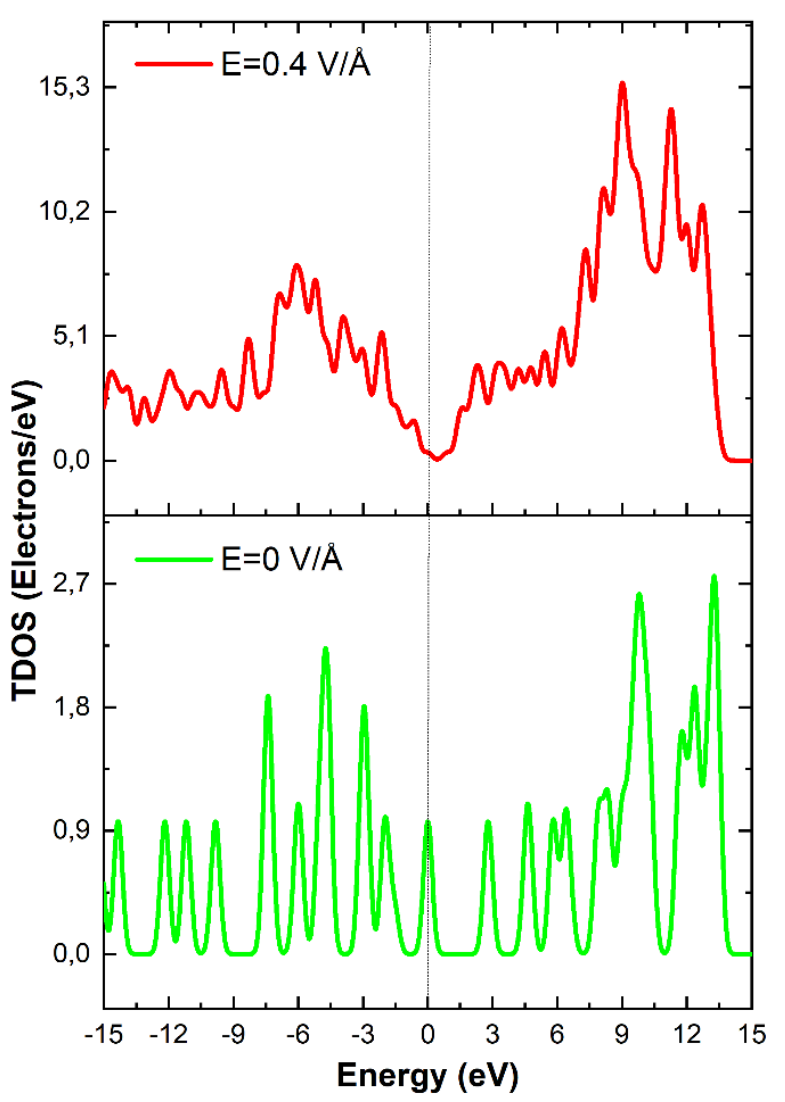

Fig .3. Calculated TDOS of graphene under $E_{\text {ext }}$ applied in z-direction.

\subsection{Optical properties}

\subsubsection{Absorption}

In this study, we studied the graphene absorption coefficient $(\alpha)$ as a function of frequency, under the applied $E_{\text {ext }}$ in z-direction in $0-8 \mathrm{eV}$ range, as shown in Fig.4. Without $E_{\text {ext }}$, the absorption spectrum consists of two peaks with different intensities. The height intensity of the first peak at $3.39 \mathrm{eV}$ and the second peak at $6.89 \mathrm{eV}$. The source of these peaks comes from two basic transitions between the electronic states of graphene. The first peak corresponds to the transition from occupied $n$ to unoccupied $n^{*}$ state in the conduction band. The second peak corresponds to the transition of $C-C$ from the $\pi-\pi^{*}$ state close to the Fermi level in the $\mathrm{sp}^{2}$ hybrid region. According to these two peaks, the graphene layer exhibits strong light absorption, which can absorb in the $U V$ and visible ranges. In the visible range, the intensity of the absorption peak decreases when $E_{\text {ext }}=0.4 \mathrm{~V} / \AA$ is applied in z-direction of graphene sheet, by reason of the increase in band gap energy.

When $E_{\text {ext }}$ is applied perpendicular to graphene layer, it causes a redshift of the absorption edge, by reason of increase the band gap energy. This behavior suggests that the $E_{\text {ext }}$ can be effectively used to adjust the graphene absorption. Additionally, through the application of $E_{\text {ext }}$ on graphene, it is possible to increase the light-absorbing capacity in the $U V$ range. According to our results of the absorption spectrum calculations, graphene possesses optical properties strongly dependent on the effect of $E_{\text {ext }}$.

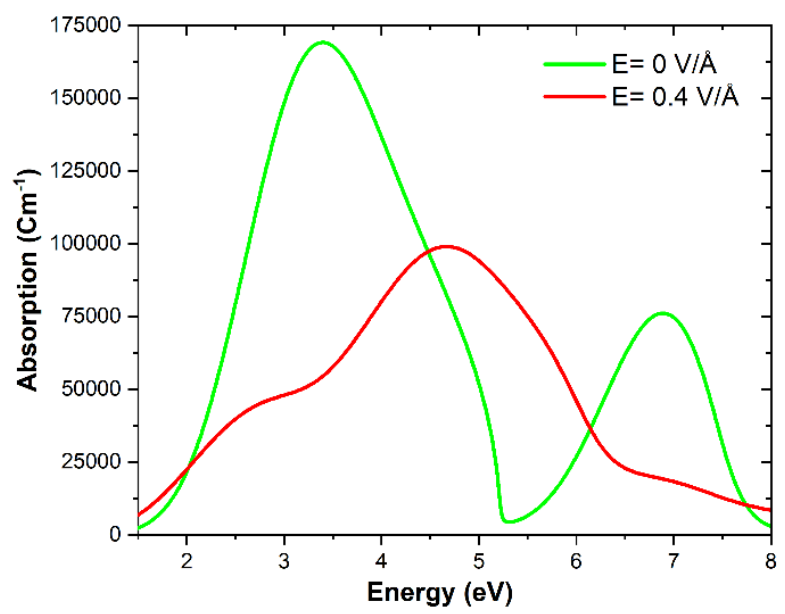

Fig. 4. Calculated absorption coefficient of graphene under $E_{\text {ext }}$ applied in z-direction.

\subsubsection{Dielectric function}

Complex frequency-dependent dielectric function, $\varepsilon(\omega)$, can be used to describe the optical properties of graphene and describe how light interacts with matter. Its determines the dispersion effects via its real part, $\varepsilon_{1}(\omega)$, and the absorption effects via its imaginary part, $\varepsilon_{2}(\omega)$ :

$$
\varepsilon(\omega)=\varepsilon_{1}(\omega)+i \varepsilon_{2}(\omega)
$$

As shown in Fig.5, the $\varepsilon_{1}(\omega)$ and $\varepsilon_{2}(\omega)$ parts are calculated under $E_{\text {ext }}$ applied perpendicularly to graphene sheet in $0-8 \mathrm{eV}$ range. In the visible range, the peaks of $\varepsilon_{1}(\omega)$ decrease under the effect of $E_{\text {ext }}$. In addition, the peaks of $\varepsilon_{2}(\omega)$ increase in the $U V$ range and decrease in the visible range, by reason of increases $E_{\text {ext }}$ from $0 \mathrm{~V} / \AA$ to $0.4 \mathrm{~V} / \AA$. These results demonstrate the exceptional ability of graphene material to absorb photons in the $U V$ region when the $E_{\text {ext }}$ is applied in zdirection. The $\varepsilon_{2}(\omega)$ part has two peaks in the $2-8 \mathrm{eV}$ range, which are always related to electron excitation, and when incident photon energy is less than $1.5 \mathrm{eV}$, it has a low value. It is important to note that the value of $\varepsilon_{1}(\omega)>0$ and $\varepsilon_{2}(\omega)=0$ in the $0-1 \mathrm{eV}$ range, which indicate the transparent range. This means that there is no absorption at low energy, because the electrons in the graphene sheet cannot react to the $E_{\text {ext }}$. Also, transitions from the maximum of the valance band to the minimum of the conduction band are forbidden. Thus, the effect of 
$E_{\text {ext }}$ can make graphene an absorbent material for a wide spectrum of energy, which means that this material can be an important component of many optoelectronic devices.
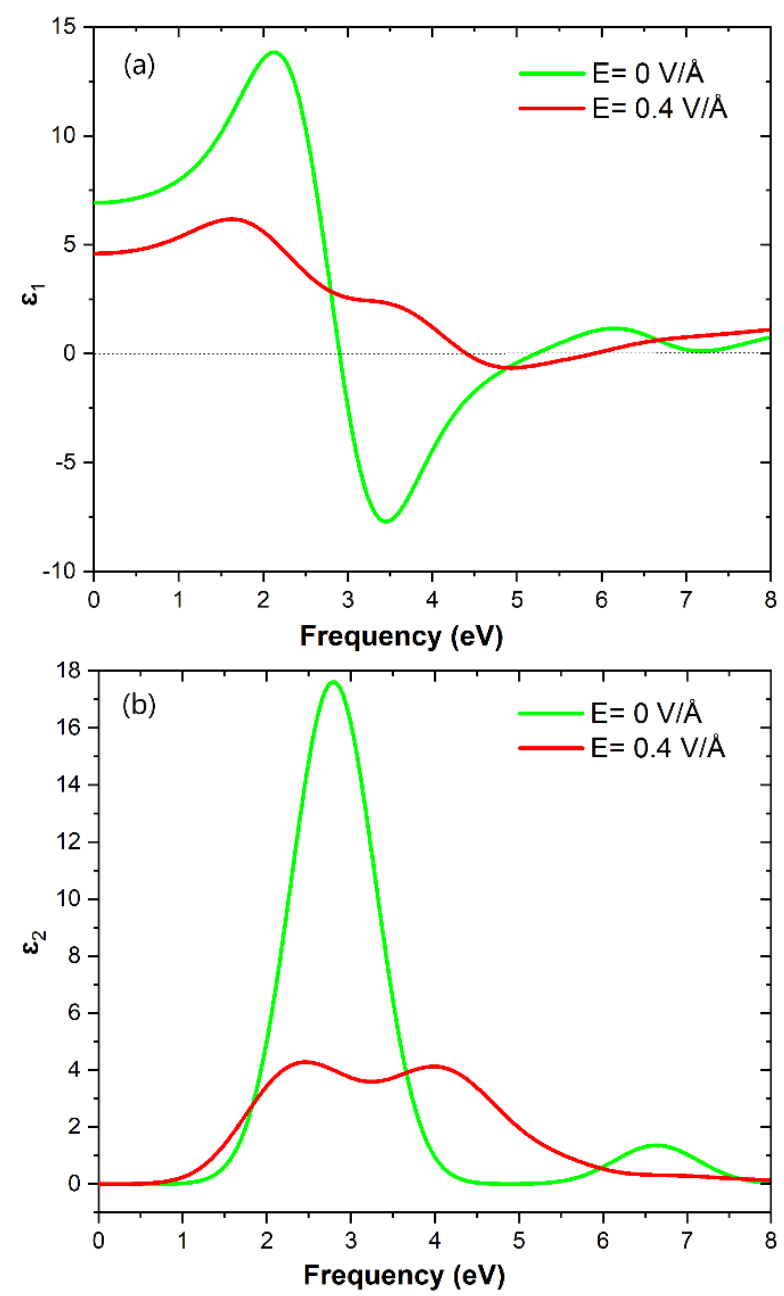

Fig. 5. Graphene dielectric function under external electric field applied in z-direction.

\subsubsection{Refractive index}

The propagation of light in absorbing materials can be considered in terms of a complex representation of the refractive index $n^{*}(\omega)$. The imaginary part $k(\omega)$, then deals with attenuation, while the real part $n(\omega)$, deals with refraction [12].

With

$$
n^{*}(\omega)=n(\omega)+i k(\omega)
$$

$$
\begin{aligned}
& n(\omega)=\sqrt{\frac{|\varepsilon(\omega)|+\varepsilon_{1}(\omega)}{2}} \\
& k(\omega)=\sqrt{\frac{|\varepsilon(\omega)|-\varepsilon_{1}(\omega)}{2}}
\end{aligned}
$$

The changes in the $n(\omega)$ and $k(\omega)$ parts of graphene layer under $E_{\text {ext }}=0.4 \mathrm{~V} / \AA$ applied in z-direction, are found using the CASTEP code, as shown in Fig. 6. The above values of static dielectric constant $\varepsilon_{1}(0)$ and static refractive index $\mathrm{n}(0)$ verify the relationship $n=\sqrt{\varepsilon_{1}}$ Tab. 2. When $E_{\text {ext }}$ is applied on graphene sheet, the $\mathrm{n}(\omega)$ part changes as a function of frequency. Therefore, graphene material is a dispersive medium, and its dispersion is affected by $E_{\text {ext }}$. The $n(\omega)$ part is greater than 0.00347 for photon energy $h v=4.86656 \mathrm{eV}$, by reason of photons incident inside graphene material are slowed down by the interaction with electrons under the effect of the $E_{\text {ext }}$. The refractive index decreases in visible range, and increases in UV range, because the application of $E_{\text {ext }}$ causes the interaction and collision between incident photons and electrons inside graphene layer. In addition, the $k(\omega)$ part is reduced in visible range. When we analyze the graphs of $\varepsilon_{2}(\omega)$ and $k(\omega)$, similar physical behaviors are observed in Fig. 5 and 6. Its two physical quantities give information of absorption light by graphene structure.
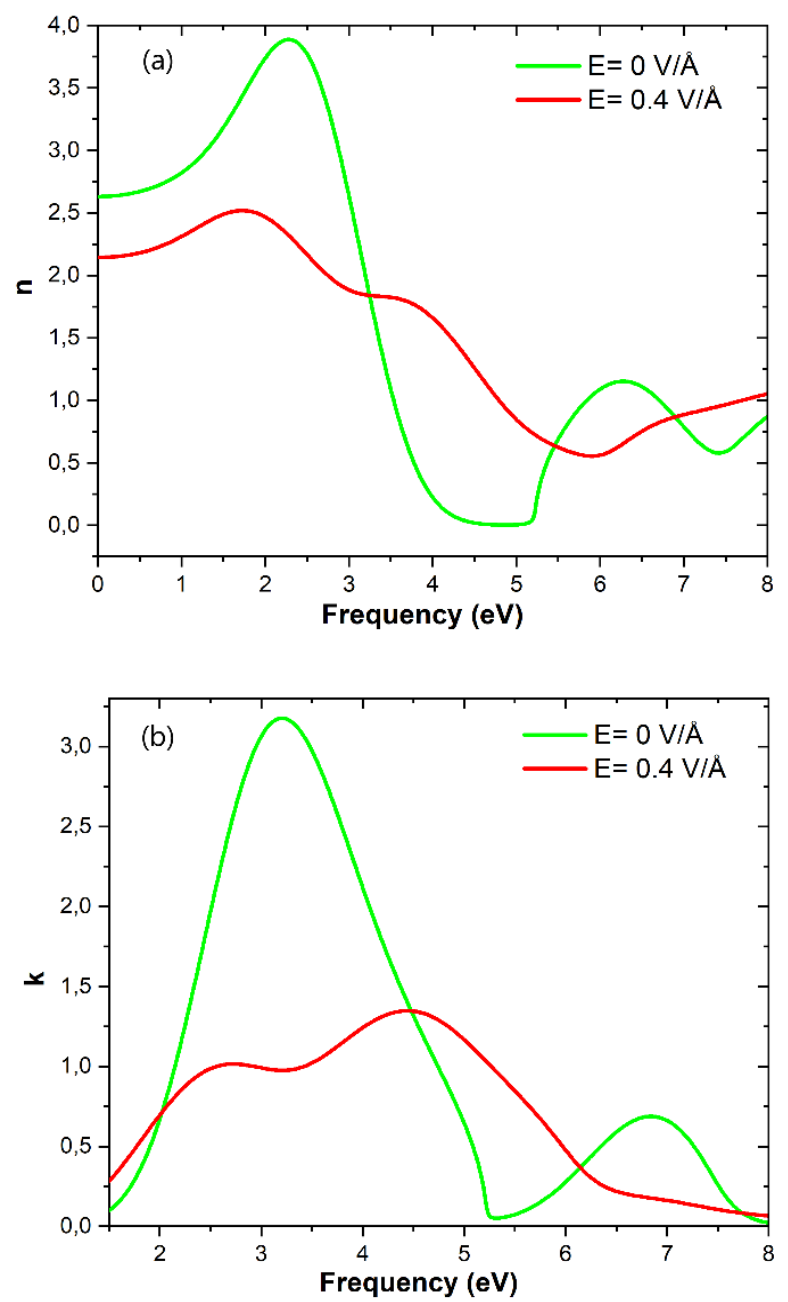

Fig. 6. Graphene complex refractive index under external electric field applied in z-direction.

Tab. 2 The static dielectric constant $\varepsilon_{1}(0)$ and the static refractive index $\mathrm{n}(0)$ of graphene under $E_{\text {ext }}$ applied in zdirection.

\begin{tabular}{|c|c|c|}
\hline Parameters & \multicolumn{2}{|c|}{$\mathrm{E}_{\mathrm{z}}$} \\
\hline $\mathrm{E}(\mathrm{V} / \AA)$ & 0 & 0.4 \\
\hline $\mathrm{n}(0)$ & 2.630 & 2.143 \\
\hline$\varepsilon_{1}(0)$ & 6.920 & 4.596 \\
\hline
\end{tabular}




\section{Conclusion}

In summary, we have studied the electronic and optical properties of graphene structure, under $E_{\text {ext }}$ applied in vertical direction using DFT calculations. The application of $E_{\text {ext }}$ has produced different changes in the band structure and optical properties of graphene layer. We have proved that the structure is a semiconductor, and the band gap energy can be significantly controlled by the influence of $E_{\text {ext }}$. We observe a change in the dielectric function, which is due to the increase in band gap energy by reason of the effect of $E_{\text {ext }}$. By applying $E_{\text {ext }}$ in z-direction, the intensity of absorption peak decreases. These results provide the basis for many applications of graphene in optoelectronic devices.

\section{References}

[1] R. Balu, X. Zhong, R. Pandey and S.P. Karna, Appl. Phys Lett 100, 052104 (2012).

[2] V. Lukose, R. Shankar and G. Baskaran, Phys. Rev. Lett. 98, 116802 (2007).

[3] J.A. Yanand M.Y. Chou, Phys. Rev. B 82, 125403 (2010).

[4] Y. Li, J. Chen and C. Zhao. RSC Adv. 7, 5667656681 (2017).

[5] N.V. Tepliakov, Q. Wu and O.V. Yazyev, Nano Letters June 11, (2021).

[6] B. Delley and J. Chern. Phys., Vol. 92, No.1, 1 January (1990).

[7] ER. McNellis, J. Meyer, and K. Reuter, Phys. Rev. B, 12 Sep (2009).

[8] J.P. Perdew, K. Burke and M. Ernzerhof, Phys. Rev. Lett, 28 OCTOBER (1996).

[9] H.J Monkhorst and J.D Pack, Phys. Rev. B, Vol. 13, No. 12, 15 June (1976).

[10] B. Mohan, A. Kumar and P.K. Ahluwalia, Physica E, 44 1670-1674 (2012).

[11] V.M. Silkin, E. Kogan and G. Gumbs, Nanomaterials, 11, 1561 (2021).

[12] H. Lashgari, A. Boochani, A. Shekaari, S. Solaymani, E. Sartipi and R.T. Mendi, Applied Surface Science, 369, 76-81 (2016). 